\title{
Apathy and Manifestations of a Wide Variety of Neuropsychiatric Disorders in Patients with Parkinson's Disease.
}

\author{
Elcin Huseyn \\ Azerbaijan State Oil and Industry \\ University, Baku, Azerbaijan \\ elcin.huseyn@asoiu.edu.az
}

\begin{abstract}
Apathy in patients with Parkinson's disease (PD) is one of the least studied manifestations of a wide range of neuropsychiatric disorders (PND). The frequency of apathy in $\mathrm{PD}$, according to various researchers, is $17-80 \%$. Structural and neurochemical changes associated with PD are considered as the leading pathophysiological factors of apathy. Possible general pathophysiological mechanisms of apathy and hypokinesia, depression, executive (frontal) cognitive functions, sleep disorders in PD are discussed. The basis of the pathophysiological commonality of apathy, hypokinesia, and executive functions is probably bilateral disturbances in the functional connections of the stratum and dorsolateral, medial parts of the prefrontal cortex. The combination of apathy and depression in PD may be due to dysfunction of the structures of the limbic system and the medial orbital prefrontal cortex responsible for motivational determined behavior. The variability of the relationship between apathy and hypokinesia, depression, cognitive impairments, sleep disorders at different stages of PD may be associated with the phenomenological heterogeneity of apathy.

Apathy worsens the quality of life, makes a significant contribution to disorders of both household and social adaptation of PD patients. It is promising to study the possibility of correcting apathy using dopaminergic therapy. Against the background of the appointment of pramipexole (1.5-3.0 mg / day) to 20 patients with PD (middle stage, according to Hen-Yar-2.5) to correct movement disorders after 4-6 weeks of therapy, a statistically significant positive dynamics of apathy were noted. According to the total assessment of the scale of apathy SE Starkstein (AS). As a result of the study, no statistically significant correlation was found between the dynamics of the total apathy indicator and the dynamics of motor symptoms of PD according to the unified rating scale for assessing PD ("Motor functions"), which, we believe, indicates an independent effect of pramipexole therapy in relation to motivational disorders.
\end{abstract}

Keywords: Parkinson's disease; apathy; pathophysiology; dopaminergic therapy; pramipexole.

\section{Introduction}

One of the leading areas of research in Parkinson's disease (PD) is the clarification of the factors that worsen the quality of life of patients. A promising task is the development of individualized therapy, considering the leading maladaptive manifestations of the disease. In this regard, neuropsychiatric disorders (PND), emotional, cognitive, behavioral disorders, fatigue, sleep, and wakefulness disorders are being actively studied. Interest in the problem of the SNP is due to their high frequency in PD, as well as a significant negative impact on the quality of life of both patients and their loved ones [1-5].

Potential difficulties in diagnosis and assessment of the phenomenology of several disorders are associated with a high comorbidity of individual clinical manifestations of SNP in PD [6]. The least studied aspect of SNP in PD is apathy. Apathy (from the Greek apatheia - dispassion) is one of the basic concepts of ethics of the Stoic philosophers, which consists in striving for the complete 1 | P a g e 
liberation of the soul from all passions. In the clinical context, apathy is seen as a violation of motivation and is characterized by a limitation of purposeful behavior, cognitive activity, flattening of emotional reactions, and a narrowing of the range of interests [7]. Apathy is observed in the clinical picture of cerebrovascular disorders, several neurodegenerative diseases - Alzheimer's disease (AD), dementia with Lewy bodies, frontal-temporal dementia, progressive supranuclear palsy, and Huntington's disease.

The diagnostic criteria for apathy used in neurological practice were proposed by R.S. Marin et al. [8] And subsequently modified by S.E. Starkstein et al. [Nine]. In 2009, these criteria were supplemented and then validated on populations of patients with various neuropsychiatric diseases by an international team of specialists. These criteria are based on the diagnosis of decreased motivation (for $\geqslant 4$ weeks) along with the presence of at least 2 of 3 characteristics of apathy: limitation of purposeful behavior, decreased cognitive activity, emotional reactions (to both positive and negative stimuli). Symptoms of apathy should reach the degree of clinical significance in violation of any aspect of the patient's daily activity, should not be explained by changes in the physical or mental state (visual impairment, hearing impairment, movement disorders, the effect of pharmacotherapy, etc.) [10]. The results of apathy diagnostics according to these criteria in the population of patients with PD were awfully close to the results of the examination on the Lily's PD Apathy Scale (LARS) [11], as well as the apathy subsection in the multisymptomatic neuropsychiatric questionnaire (NPI) by J. Cummings et al. ... In addition to these scales, according to the recommendations of the Association for Movement Disorders in 2008, for the diagnosis of apathy in PD, it is proposed to use the apathy scale developed by S.E. Starkstein et al. (AS) [12].

According to observational studies [13], apathy is observed in $17-80 \%$ of patients with PD. Such a large spread is probably due to the use of different methods for assessing the phenomenon and the heterogeneity of patient groups. According to a Norwegian population-based study, apathy was noted in $38 \%$ of patients. Moreover, in 5\% of cases, this symptom occurred as an independent phenomenon, regardless of the presence of depression, mild cognitive impairment or dementia, the use of antidepressants and antipsychotic drugs [14]. In a recent study of the incidence of apathy using new amended criteria, it was revealed in $27 \%$ of cases [15]. Similar data were obtained in our clinical study: the incidence of apathy in PD patients without dementia was $25 \%$ [16].

It was found that the incidence of apathy in PD patients significantly exceeds its incidence in the corresponding age population. Thus, in one comparative study, apathy was observed in $40-44 \%$ of patients with PD and was absent in the control group [17]. In a recent study by L.L. Jordan et al. [18] apathy was detected in $44 \%$ of patients with PD and in $20 \%$ in the comparison age group. Apathy in PD is noted more often than in other chronic disease - osteoarthritis, compared to PD in limiting motor activity [19]. Thus, apathy cannot be explained only by age factors or a psychological reaction to a chronic illness, but is a manifestation of morpho functional and neurochemical changes in PD.

\section{Materials and Methods}

The role of dysfunction of the basal ganglia in the pathophysiology of apathy was revealed in the observation of patients with bilateral lesions of the shell, caudate nucleus, Globus pallidus, mediodorsal portions of the optic hillock [20]. It is assumed that the motivational components of behavior are regulated by dopaminergic projections connecting the ventral operculum, the ventral part of the Globus pallidus, with the mesolimbic and prefrontal mesocortical structures of the brain. The nucleus accumbens ( $\mathrm{n}$. Accumbence) is considered one of the key structures that ensure the 
functional interaction of these parts of the brain and the modulation of purposeful behavior [21]. Using the methods of functional neuroimaging in patients with asthma, the relationship between apathy and dysfunction of the prefrontal medial-orbital cortex, the anterior cingulate gyrus, has been shown [13]. A recent neuroimaging study using voxel-oriented morphometry showed a correlation between the severity of apathy in PD with a decrease in the gray matter density of the precentral gyrus, the lower frontal and parietal lobes of the brain, and the right posterior cingulate gyrus [22]. In the development of apathy and bradyphrenia, it also assumes the role of a violation of the connection between the inner segment of the Globus pallidus and the pedunculopontine nucleus (PPN) of the brain stem [23].

Questions about the phenomenological independence of apathy in PD patients are closely related to the assessment of its relationship with depression. On the one hand, apathy is a symptom and an additional criterion of depression, on the other hand, it is detected in patients with PD and without key signs of depression (feelings of sadness, anhedonia), differing from depression by emotional flattening [24]. In PD, a high degree of correlation between these disorders was noted. In a study of patients with $\mathrm{PD}, \mathrm{AD}$, progressive supranuclear palsy, and frontotemporal dementia, a correlation between depression and apathy was revealed only in PD [24].

For the differential diagnosis of apathy and depression, the results of a recent study that clarify the relationship between apathy and anhedonia in PD are of interest. It has been shown that anhedonia in patients with apathy is more represented by a decrease in motivation to receive pleasure along with maintaining an adequate emotional response to positive events [18].

In PD, as in other neuropsychiatric diseases, an association of apathy and cognitive (dementia and pre-dementia) impairments has been revealed. Manifestations of apathy, along with slow thinking, impaired attention was described in the framework of the symptom complex "bradyphrenia" by F. Novel in 1922. Modern studies reveal a connection between apathy and disorders of frontal executive functions, including considering the temporal parameters of cognitive activity, which allows us to discuss the conjugation and bradyphrenia [12].

The relationship between apathy and the severity of PD motor symptoms has not been adequately studied. An association of apathy (along with concomitant depression and cognitive impairment) with a more severe stage of PD and a predominantly kinetic rheumatic form of the disease has been shown [25]. Among the independent risk factors for apathy, the rapid progression of speech disorders, axial symptoms (postural disturbances, postural instability) are also indicated [26].

Our studies have confirmed the high comorbidity of apathy and depression. So, only in $20 \%$ of cases (i.e., in $5 \%$ of the surveyed) apathy occurred separately from depression [16]. There was a statistically significant positive correlation between apathy and depression in the 2nd and 3rd stages of PD, along with its absence in the 1st and 4th stages. The difference in the relationship between apathy and depression at different stages of PD may reflect the phenomenological heterogeneity of apathy, i.e., it can be considered as a component of a depressive disorder, a comorbid symptom, or an independent manifestation of the disease. The results of cluster analysis, which showed that apathy and depression belong to different groups of signs, are a more powerful argument in favor of the syndromes autonomy of apathy [16]. The combination of apathy and depression in PD may be due to common links in the pathophysiology of these disorders - dysfunction of the structures of the limbic system and the medial orbital prefrontal cortex responsible for motivational conditioned behavior (the so-called reward processes). To the brain systems "Rewards" refer to dopaminergic projections connecting the ventral tectum with the mesolimbic and mesocortical regions of the brain 
[21].

The results of our study confirmed the relationship between apathy and impairment of executive (frontal) functions in PD patients without dementia with stage 2 PD. A decrease in executive functions is associated with violations of purposeful behavior, a decrease in independent activity due to a disorder in the mechanisms for developing an action plan, switching from one activity to another, etc. Lateral and medial orbital prefrontal cortex [16].

Of interest are our data on the relationship between apathy and sleep disorders [16]. In addition to the possible negative impact of poor sleep quality on the emotional state of patients and the general level of brain activation during wakefulness (decrease), it can be assumed that there are common pathophysiological components of apathy and dyssomnia. One of these common mechanisms is PPJ dysfunction due to degeneration of its neurons in PD and impaired connections with the basal ganglia and other nuclei of the trunk. PPN plays an important role in modulating rapid eye movement (REM) sleep. PPJ dysfunction is associated with the development of one of the variants of Parasomnia-behavior disorder syndrome in the SDH phase (SDS SBDH). Previously, we found a positive correlation between the clinical manifestations of RLS RRH and apathy [27]. Considering the data from several studies that allow us to consider SNP SBDH as a risk factor for the progression of cognitive impairment [28], it can be assumed that apathy, cognitive impairment, and sleep disorders are manifestations of a single complex of the SNP.

To clarify the pathophysiology of apathy in PD, the relationship between apathy and hypokinesia revealed in our study seems to be significant [16]. It is important to recall the heterogeneity of the symptom of hypokinesia and its components. Traditionally, hypokinesia is considered as the leading motor symptom of parkinsonism. In the classification of hypokinesia in PD N. Narabayashi [29], this variant is called primary hypokinesia. According to modern concepts, primary hypokinesia can be associated with dysfunction of the cortical-subcortical ring and dissociation of the basal ganglia and the premotor cortex. The severity of the motor aspects of hypokinesia (slowing down of the pace of movements, difficulty in initiating voluntary movements, decrease in the amplitude of movements, etc.) Decreases with dopaminergic replacement therapy. There is also the so-called psychomotor hypokinesia, manifested by the lack of motivation to action, the initiation of motor programs [29]. In PD, hypokinesia can be caused by both components. The basis of the pathophysiological commonality of apathy and hypokinesia is probably bilateral disturbances in the functional connections of the stratum and dorsolateral, medial parts of the prefrontal cortex [30].

The variability of the relationship between apathy and hypokinesia, depression, cognitive impairment, sleep disorders at different stages of PD may be associated with the heterogeneity of these clinical manifestations and their severity. At different stages of PD, considering the individual characteristics of the course of the disease, the pathological process may involve individual functional subsystems of multilevel neural networks that unite the basal ganglia, structures of the limbic system, and the prefrontal cortex [29]. In this regard, it is interesting to try to classify the variants of apathy depending on the preferential localization of the pathological process. R. Levy and B. Dubois [7] distinguish three main subtypes of apathy: apathy associated with impaired emotional-affective processes; apathy associated with impaired cognitive functions, and apathy caused by impaired self-activation processes. We believe that in PD, given the diffuse nature and neurochemical multicomponent nature of the pathological process, we can only talk about the possible dominance of certain pathophysiological subtypes of apathy.

From a clinical point of view, it is important to understand the prognostic value of diagnosing apathy in PD patients. According to a prospective long-term study conducted by K. Dujardin et al. 
[31], the presence of apathy worsens the prognosis and is associated with a faster rate of progression of cognitive impairment, the development of dementia, and a decrease in daily activity. At the same time, the results of studies on the effect of apathy on the quality of life of patients are rather contradictory. In a multicenter Italian study, P. Barone et al. [32], which involved 1072 patients (mean duration of PD - 5.1 years, stage - 1.5-2.5 according to Hen-Yar), it was shown that apathy is a leading symptom associated with low self-esteem values of life. To a lesser extent, the quality of life was influenced by fatigue and cognitive impairment. According to a recent multicenter Spanish study by J. Benito-Leon et al. [33], which included 557 patients (with a PD diagnosis established no more than 2 years ago), apathy is one of the leading factors in the low assessment of the quality of life in patients already in the early stages of the disease. Along with this, in other studies, depression combined with fatigue and sleep disturbances [3], anxiety and visual hallucinations [34] was the leading SNP that worsened the quality of life [34]. The variability in these data appears to be due to the use of different methods for assessing apathy and other SNP.

According to our data [16], the total assessment of apathy is statistically significantly correlated with the indicators of daily activity (violation of everyday activities) of patients. The connection between the manifestations of apathy and the maintenance of social contacts, emotional, cognitive components of assessing the quality of life in different stages of PD was noted.

Thus, apathy undoubtedly makes a significant contribution to the disturbance of both every day and social adaptation of PD patients. According to our observations [16], the negative impact of apathy on the quality of life is revealed even in cases of mild and moderate severity of the syndrome, in connection with which the issues of therapy are truly relevant.

Approaches to the treatment of apathy in PD require further development. When apathy is combined with depression, antidepressants with an activating effect may be prescribed. However, due to the possibility of apathy on the background of the use of antidepressants - serotonin reuptake inhibitors (SSRIs; "SSRI-induced apathy") [35] - it is necessary to differentiate apathy from depression and conduct careful monitoring of such patients during therapy. In the case of apathy "isolated" from depression, antidepressants are of little promise. The effectiveness of the acetylcholinesterase inhibitor rivastigmine in apathy in patients with PD and dementia has been demonstrated [36].

Considering the putative dopaminergic mechanisms of apathy, it is of particular interest to study the efficacy of dopaminergic therapy in patients with PD and apathy. In the literature, there are isolated reports of a decrease in apathy against the background of correction of fluctuations in motor and non-motor symptoms of PD [37]. Small open-label studies have been published showing the efficacy of dopamine receptor agonists (ADRs) ropinirole and piribedil in apathy in PD patients who underwent deep stimulation of the subthalamic nucleus [38-39]. In a meta-analysis of the results of randomized controlled trials, the ADR of pramipexole of 70 patients with apathy in $63.2 \%$, receiving drug therapy, there was a decrease compared with $45 \%$ of patients who were prescribed placebo [40].

\section{Results}

The results of our small open-label study confirmed the potential efficacy of dopaminergic therapy for apathy in PD patients. Against the background of the appointment of pramipexole (1.5$3.0 \mathrm{mg} /$ day) to 20 patients with PD (middle stage - 2.5 according to Hen-Yar) after 4-6 weeks of therapy, statistically significant dynamics of the total score on the AS scale was observed, and the values of point 7 of the scale, which assesses the motivation for voluntary activity. In addition, 
against the background of therapy, there was a statistically significant dynamics of the total score of the Beck Depression Inventory, the sections of the scale characterizing manifestations of apathy (point 12 - motivation for social contacts and point 15 - motivation for voluntary activity). We did not find a statistically significant relationship between the dynamics of apathy indicators and the dynamics of motor symptoms of PD during therapy, which probably indicates an independent effect of pramipexole therapy in relation to motivational disorders.

The effect of pramipexole on apathy, as well as the antidepressant effect of the drug, established during numerous open and placebo-controlled studies, is probably due to the activation of the D3subtype of dopamine receptors in the structures of the limbic system and the prefrontal cortex. In addition to the antidepressant effect, we previously described the results of our own studies of the positive effect of pramipexole on other comorbid APN apathy in PD patients: moderate impairment of cognitive (executive) functions, fatigue, and sleep disorders [41].

Evaluation of the effectiveness of approaches to dopaminergic therapy in different variants and severity of apathy should be the subject of further clinical and pharmacological studies. Reducing apathy with the help of dopaminergic therapy will allow avoiding polypharmacotherapy with its undesirable effects and increasing patient adherence to treatment.

In addition to pharmacotherapy, to correct apathy, a complex of non-pharmacological methods can be used - psychotherapy, cognitive-behavioral therapy. However, the experience of their use, as well as other types of non-pharmacological effects, is still insufficient. Pharmacotherapy and nondrug treatments require active cooperation with the patient's relatives or caregivers. The complex of rehabilitation measures should include an increase in the motor, cognitive and social activity of patients, stimulation of interests, assistance in planning behavioral activity, and involvement in new types of activity.

\section{References}

1. Aarsland D, Marsh L, Schrag A. Neuropsychiatric symptoms of Parkinsons disease. Mov Disord. 2009; 24 (15): 2175-86. DOI: 10.1002 / MDs. 22589.

2. Scaravilli T, Gasparoli E, Rinaldi F, et al. Health-related quality of life and sleep disorders in Parkinson's disease. Neurol Sci. 2003 Oct; 24 (3): 209-10. DOI: http://dx.doi.org/10.1007/s10072003-0134-y.

3. Challenger DA, Lees AJ, Shrag A. What are the most important nonmotor symptoms in patients with Parkinsons disease and are we missing them? Mov Disord. 2010; 15 (15): 2493-500. DOI: 10.1002 / MDs. 23394.

4. Nodel MR, Yakhno NN. Neuropsychiatric disorders of Parkinson's disease. Neurology, Neuropsychiatry, psychosomatic. 2009; (2): 3-8. [Nodel 'MR, Yakhno NN. Nervous and mental disorders of Parkinson's disease. Nevrologiya, neiropsikhiatriya, psychosomatic = Neurology, Neuropsychiatry, Psychosomatics. 2009; (2): 3-8. (In Russ.)]. DOI: http://dx.doi.org/10.14412/2074-2711-2009-30.

5. Levin OS. Parkinson's disease as a neuropsychiatric disease. A guide for physicians on the materials of the II National Congress on Parkinson's Disease and Movement Disorders. Under. Ed. S.N. Illarioshkina, O.S. Levin. Moscow: NTSN RAMS; 2011. P. 99-104. [Levin OS. Bolezn 'Parkinsona kak neiropsikhiatricheskoe zabolevanie. Rukovodstvo dlya vrachei po materialam II Natsional'nogo kon- gressa po bolezni Parkinsona i rasstroistvam dvizhenii [Parkinson's illness as neuropsychiatric disease. The management for doctors on materials II of the National Congress due to illness Parkinson and to disorders of movements]. Illarioshkina SN, Levina OS, editors. Moscow: 
NTsN RAMN; 2011. P. 99-104.]

6. Voznesenskaya TG. Apathy Syndrome. Neurological journal. 2013; 18 (3): 4-10. [Voznesenskaya TG. Apathy syndrome. Nevrologicheskii Zhurnal. 2013; 18 (3): 4-10. (In Russ.)]

7. Levy R, Dubois B. Apathy, and functional anatomy of the prefrontal cortex-basal ganglia circuits. Cereb Cortex. 2006; 16 (7): 916-28. DOI: http://dx.doi.org/10.1093/cercor/bhj043. Epub 2005 Oct 5.

8. Marin RS, Biedrzycki RC, Firinciogullari S. Reliability and validity of the Apathy Evaluation Scale. Psychiatry Res. 1991; 38 (2): 143-62. DOI: http://dx.doi.org/10.1016/0165-1781 (91)90040V.

9. Starkstein SE, Jorge R, Mizrahi R, Robinson RG. A prospective longitudinal study of apathy in Alzheimers disease. J Neurol Neurosurg Psychiatr. 2006; 77 (1): 8-11. DOI: http://dx.doi.org/10.1136/jnnp.2005.069575.

10. Robert P, Onyike CU, Leentjens AF, et al. Proposed diagnostic criteria for apathy in Alzheimer's disease and other neuroposychiatric disorders. EUR Psychiatry. 2009; 24 (2): 98-104. DOI: 10.1016 / j.eurpsy.2008.09.001. Epub 2009 Feb 7.

11. Sockeel P, Dujardin K, Devos D, et.al. The Lille apathy rating scale (LARS): a new instrument for detecting and quantifying apathy: validation in Parkinson's disease. J Neurol Neurosurg Psychiatry. 2006; 77 (5): 579-84. DOI: http://dx.doi.org/10.1136/jnnp.2005.075929.

12. Starkstein SE, Mayberg HS, Preziosi TJ, et.al. Reliability, validity, and clinical correlates of apathy in Parkinsons disease. J Neuropsychiatry Clin Neurosci. 1992; 4 (2): 134-9.

13. Leroi I, David R, Robert PH. Apathy in Parkinsons disease. In: Psychiatry of in Parkinsons disease. Admire KP, O'Brien JT, Taylor J-P, editors. Basel: Karger; 2012. P. 27-40. DOI: http://dx.doi.org/10.1159/000331524.

14. Pedersen KF, Larsen JP, Alves G, Aarsland D. Prevalence and clinical correlates of apathy in Parkinsons disease: a community-based study. Parkinsonism Relat Disord. 2009 May; 15 (4): 295 9. DOI: 10.1016 / j. Paradise. 2008. 07. 006. Epub 2008 Sep 17.

15. Mulin E, Leone E, Dujardin K, et al. Diagnostic criteria for apathy in clinical practice. Int J Geriatr Psychiatry. 2011; 26 (2): 158-65. DOI: 10.1002 / GPs. 2508.

16. Nodel MR, Yakhno NN. Apathy in Parkinson's disease. Neurological journal. 2014; 1: 9-15. [Nodel of MR, Yakhno NN. Apathy at Parkinson's illness. Nevrologicheskii Zhurnal. 2014; 1: 9-15. (In Russ.)]

17. Bogart KR. Is apathy a valid and meaningful symptom or syndrome in Parkinsons disease? A critical review. Health Psychol. 2011; 30 (4): 386-400. DOI: 10.1037 / a0022851.

18. Jordan LL, Zahodne LB, Okun MS, Bowers D. Hedonistic and behavioral deficits associated with apathy in Parkinsons disease: potential treatment implications. Mov Disord. 2013; 28 (9): 1301-04. DOI: 10.1002 / MDs. 25496. Epub 2013 May 27.

19. Pluck GC, Brown RG. Apathy in Parkinson's disease. J Neurol Neurosurg Psychiatry. 2002; 73 (6): 636-42. DOI: http://dx.doi.org/10.1136/jnnp.73.6.636.

20. Czernecki V, Schupbach M, Yaici S, et al. Apathy following subthalamic stimulation in Parkinson disease: a dopamine responsive symp- tom. Mov Disord. 2008; 23 (7): 964-9. DOI: 10.1002 / MDs. 21949.

21. Robbins T, Evritt B. Neurobehavioral mechanism of reward and motivation. Curr Opin Neurobiol. 1996; 6 (2): 228-36. DOI: http://dx.doi.org/10.1016/S0959- 4388 (96) 80077-8.

22. Reijnders JS, Scholtissen B, Weber WE, et al. Neuroanatomical correlates of apathy in Parkinson's disease: a magnetic resonance imaging study using fixed-based morphometry. Mov 
Disord. 2010; 25 (14): 2318-25. DOI: 10.1002 / MDs. 23268.

23. Starkstein SE, Federoff JP, Price TR, et al. Apathy following cerebrovascular lesions. Stroke. 1993: 24 (11): 1625-30. DOI: http://dx.doi.org/10.1161/01.STR.24.11.1625.

24. Levy ML, Cummings JL, Fairbanks LA, et al. Apathy is not depression. J Neuropsychiatr Clin Neuroscie. 1998; 10 (3): 314-9.

25. Reijnders J, Ehrt U, Lousberg R, et al. The association between motor subtypes and psychopathology in Parkinsons disease. Parkinsonism Relat Disord. 2009; 15 (5): 379-82. DOI: 10.1016 / j. Paradise. 2008. 09. 003. Epub 2008 Oct 31.

26. Pedersen KF, Alves G, Aarsland D, et al. Occurrence and risk factors for apathy in Parkinsons disease: a 4-year prospective longitudinal study. J Neurol Neurosurg Psychiatry. 2009; 80 (11): 1279-82. DOI: 10.1136 / jnnp. 2008. 170043.

27. Nodel MR, Rusakova IM, Yakhno NN. Clinical assessment of sleep and wakefulness disorders in Parkinson's disease. Neurological journal. 2010; (2): 19-25. [Nodel 'MR, Rusakova IM, Yakhno NN. Clinical assessment of violations of a dream and wakefulness at Parkinson's illness. Nevrologicheskii Zhurnal. 2010; (2): 19-25. (In Russ.)]

28. Vendette M, Gagnon JF, Decary A, et al. REM sleep behavior disorder predicts cognitive impairment in Parkinson disease without dementia. Neurology. 2007; 69 (19): 1843-9. DOI: http://dx.doi.org/10.1212/01.wnl. 0000278114.14096.74.

29. Narabayashi H. Akinesia in parkinsonism. In: Movement disorders in Neurology and Neuropsychiatry. Joseph AB, Young RR, editors. Oxford: Blackwell Science; 1999. P. 185-8.

30. Tekin S, Cummings JL. Frontal-subcortical neuronal circuits and clinical psychiatry: an update. J Psychosom Res. 2002; 53 (20: 647-54.

31. Dujardin K, Sockeel P, Delliaux M, et al. Apathy may herald cognitive decline and dementia in Parkinsons disease. Mov Disord. 2009; 24 (16): 2391-7. DOI: 10.1002 / MDs. 22843.

32. Barone P, Antonini A, Colosimo C, et al. The Priamo Study: a multicenter assessment of nonmotor symptoms and their impact on quality of life in Parkinsons disease. Mov Disord. 2009; 11 (24): 1641-9. DOI: http://dx.doi.org/10.1002/mds.23872.

33. Benito-Leon J, Cubo E, Coronell C, et al. Impact of apathy on health-related quality of life in recently diagnosed Parkinsons disease: the ANIMO study. Mov Disord. 2012; 27 (2): 211-8. DOI: 10.1002 / MDs. 23872. Epub 2011 Jul 20.

34. MC Kinlay A, Grace RC, Dalrymple-Alford JC, et al. A profile of neuropsychiatric problems and their relationship to quality of life for Parkinsons disease. Parkinsonism Relat Disord. 2008; 14 (1): 37-42. DOI: http://dx.doi.org/10.1016/j.parkreldis.2007.05.009. Epub 2007 Jul 12

35. Wangpakaran N, van Reekum R, Wangpakaran T, Clarke D. Selective serotonin reuptake inhibitor use associated with apathy among depressed elderly: a case control study. Ann Gen Psychiatry. 2007; 6: 7. DOI: http://dx.doi.org/10.1186/1744-859X-6-7.

36. Aarsland D, Mosimann UP, McKeith IG. Role of cholinesterase inhibitors in Parkinsons disease and dementia with Lewy bodies. J Geriatr Psychiatry. 2000; 15 (5): 387-92.

37. Campbell JJ, Duffy JD. Treatment strategies in motivating patients. Psychiatry Ann. 1997; 27: 44-9.

38. Czernecki V, Schupbach M, Levy R, et al. Apathy following subthalamic nucleus stimulation in Parkinsons disease is improved by treatment with a dopaminergic agonist. Mov Disord. 2006; 21 : 652.

39. Thobois S, Lhommee E, Klinger $\mathrm{H}$, et al. Parkinsonian apathy responds to dopaminergic stimulation of D2 / D3 receptors with piribedil. Brain. 2013; 136 (PT 5): 1568-77. Do: 10.1093 / 
brain / awt067. Epub 2013 Mar 29.

40. Leentjens A, Koester J, Fruh B, et al. The effect of pramipexole on mood and motivational symptoms in Parkinson disease: a meta-analysis of placebo-controlled studies. Clin Ther. 2009; 31 (1): 89-92. DOI: 10.1016 / j. Cleanser. 2009. 01. 012.

41. Nodel MR, Yakhno NN. Mirapex (pramipexol) in the treatment of non-motor disorders in Parkinson's disease. Journal of Neurology and Psychiatry. S.S. Korsakov. 2008; 108 (5): 32-8. [Nodel MR, Yakhno NN. Mirapex (pramipexole) in the treatment of non-motor disturbances in Parkinson's disease. Zhurnal nevrologii I psychiatry, I'm. S.S. Korsakova. 2008; 108 (5): 32-8. (In Russ.)] 